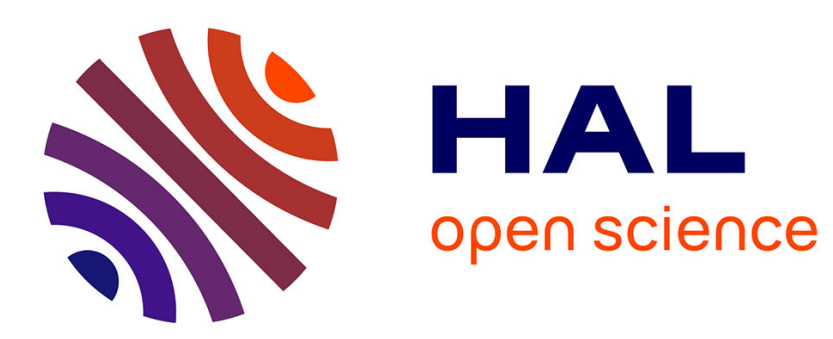

\title{
The role of thin films in wetting
}

Abraham Marmur

\section{To cite this version:}

Abraham Marmur. The role of thin films in wetting. Revue de Physique Appliquée, 1988, 23 (6), pp.1039-1045. 10.1051/rphysap:019880023060103900 . jpa-00245906

\section{HAL Id: jpa-00245906 https://hal.science/jpa-00245906}

Submitted on 1 Jan 1988

HAL is a multi-disciplinary open access archive for the deposit and dissemination of scientific research documents, whether they are published or not. The documents may come from teaching and research institutions in France or abroad, or from public or private research centers.
L'archive ouverte pluridisciplinaire HAL, est destinée au dépôt et à la diffusion de documents scientifiques de niveau recherche, publiés ou non, émanant des établissements d'enseignement et de recherche français ou étrangers, des laboratoires publics ou privés. 
Classification

Physics Abstracts

$68.10-68.15-68.40-68.45$

\title{
The role of thin films in wetting
}

\author{
Abraham Marmur \\ Department of Chemical Engineering, Technion - Israel Institute of Technology, 32000 Haifa, Israel
}

(Reçu le 28 septembre 1987, révisé le 21 décembre 1987, accepté le 22 décembre 1987)

\begin{abstract}
Résumé. - Nous discutons le rôle des films minces dans les phénomènes de mouillage. Nous distinguons trois modes d'étalement spontané : mouillage total, mouillage partiel et un mode mixte. Un film mince peut avoir une épaisseur qui est soit dans le domaine de taille moléculaire soit dans le domaine colloïdal. Les films moléculaires adsorbés sont surtout associés aux situations de mouillage partiel. Les films colloïdaux se forment à partir du liquide en volume dans des situations dynamiques de mouillage total. Leur existence à l'équilibre dépend de l'orientation dans le champ gravitationnel. L'étalement des mélanges conduit à une phénoménologie complexe qui n'est pas encore décrite par des modèles théoriques. Les différences entre les tensions de surface, le caractère plus ou moins volatil et la tendance à l'adsorption des divers composants contrôlent les propriétés de mouillage qualitativement et quantitativement.
\end{abstract}

\begin{abstract}
The role of thin films in wetting is reviewed. Three modes of spontaneous spreading are discussed : incomplete spreading, complete spreading and mixed-mode spreading. A thin film can be either molecular or colloidal in thickness. Molecularly adsorbed films are mainly associated with incomplete spreading. Colloidal films usually extend from the bulk of the liquid in dynamic situations of complete spreading. Their existence at equilibriuim with the bulk depends on the orientation in the gravitational field. The spreading of mixtures involves complex phenomenology, much of which is yet unaccounted for by theory. The differences in surface tensions, volatilities and tendencies for adsorption among the components determine the quantitative as well as qualitative features of the spreading.
\end{abstract}

\section{Introduction.}

Wetting of solid surfaces has had an important role in many technological developments since ancient times. Whenever a surface is painted, an adhesive is applied, an insecticide is spread or an ink is used, good or controlled wetting is required. Considering the many applications of wetting in daily life, biology, medicine and various industrial processes, it is surprising to realize how slow has the advancement in understanding wetting been. Many important observations were made during the nineteenth century and the early years of this century. However, a deeper theoretical understanding of wetting has been developed mostly in recent years, and much still awaits explanation.

Wetting phenomena may be classified as spontaneous or forced. The former refers to the spreading of a liquid on a solid surface toward a thermodynamic equilibrium. The latter describes a situation where the solid-liquid interfacial area is forcibly increased beyond equilibrium, as, for example, in painting with a brush. The liquid is then prevented from returning to equilibrium, usually by increasing its viscosity through polymerization and/or evaporation of a solvent. In this paper, only spontaneous wetting will be discussed. In addition, spreading of a liquid on a solid may be classified as complete or incomplete. The latter implies a non-zero equilibrium contact angle, while the former implies that the final stage is a thin liquid film spread on the solid surface.

The role of thin films in wetting is by no means limited to the final stage. It was recognized long ago [1] that a thin film is moving ahead of a completely spreading drop. In addition, it was realized that films which are adsorbed onto the solid, probably from the vapor, may control the extent of wetting $[1,2]$. To avoid semantic confusion, films of molecular thickness will be called adsorbed films, while films of colloidal thickness will be referred to as colloidal films. The term thin films will be used in the generic sense.

The relationship between thin films and wetting is very complex. Many aspects, especially those related 
to the spreading of mixtures, are not yet well understood. The present paper reviews the current knowledge of the role of thin films in wetting and points out some of the open questions. For other aspects of wetting, the reader is referred to previous review articles [3-5].

\section{Incomplete spreading.}

Incomplete spreading is characterized by a non-zero contact angle, i.e. the equilibrium state of the liquid is not a thin film. However, a thin film may extend from the bulk of the liquid. The following discussion analyzes this possibility and its implications.

2.1 The Young EQuation. - The basic thermodynamic equation which determines the equilibrium state of a wetting system was published in 1805 by Young [6] :

$$
\cos \theta=\frac{\gamma_{\mathrm{sv}}-\gamma_{\mathrm{sl}}}{\gamma_{\mathrm{lv}}}
$$

where $\theta$ is the contact angle, as shown in figure 1a, and $\gamma_{\mathrm{sv}}, \gamma_{\mathrm{sl}}, \gamma_{\mathrm{lv}}$ are the interfacial tensions of the solid-vapor, solid-liquid and liquid-vapor interfaces, respectively. This equation has been derived by a few methods [7], of various degrees of rigor. All methods lead to the same equation, however differ in the insight they provide. A recent detailed deri-

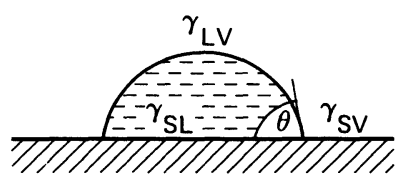

(a)

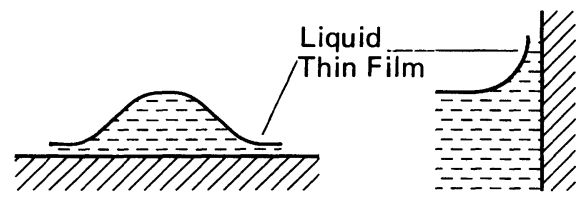

(b)

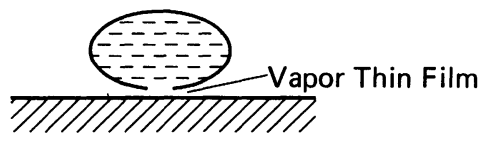

(c)

Fig. 1. - (a) The definition of the contact angle. (b) An exaggerated view of a thin film extending from the bulk of the liquid for low apparent contact angles. (c) An exaggerated view of a thin film extending from the bulk of the vapor for a high apparent contact angle. vation [4] has emphasized the following features related to the Young equation :

(1) $\theta$ is the local contact angle at any given point on the contact line. The Young equation is therefore locally valid for any surface, whether rough or smooth, heterogeneous or homogeneous. However, it should be remembered that this equation is based on the assumption that the solid is nondeformable and insoluble.

(2) The Young equation is correct only if the interfacial tensions are regarded as local quantities at the contact line region. These interfacial tensions are different from the interfacial tensions far away from the contact line, because of the effect of the third phase on the energy of the interface between the other two.

These conclusions put the Young equation in a unique position : it is theoretically correct, but none of the parameters in it can be experimentally measured. This is so, because there does not exist yet a method to measure the interfacial tensions which involve the solid; the liquid-vapor interfacial tension can be measured only far away from any solid surface ; and also the local contact angle (at the contact line itself) is not yet accessible to direct measurement. Thus, although the trends predicted by equation (1) have been observed for many years, a direct verification of it has eluded scientists so far.

2.2 THE EFFECT OF AN ADSORBED FILM. - The solid-vapor interfacial tension which appears in the Young equation, $\gamma_{\mathrm{sv}}$, refers to the solid surface as seen by the liquid drop at equilibrium. Since equilibrium should prevail everywhere in the system, it is conceivable that vapor may absorb on the bare solid. This would imply the following modification in the solid-vapor interfacial tension, as first realized by Bangham and Razouk [8] :

$$
\gamma_{\mathrm{sv}}=\gamma_{\mathrm{s}}-\pi^{0}
$$

where $\gamma_{\mathrm{s}}$ is the interfacial tension of the solid in vacuum. The film pressure, $\pi^{0}$, is defined by this equation as the reduction in the surface energy of the solid due to adsorption. Equations (1) and (2) then imply that the contact angle is increased by the occurrence of adsorption.

There is a large body of experimental information that may be explained by the film pressure concept. It was realized already in the early experiments on wetting that some liquids, which had been expected to completely spread on a solid surface, did not do it. Hardy [1] noticed that pure acetic acid or tripropylamine did not completely spread on clean glass in dry air. He was able to show, by demonstrating a reduction in the static friction coefficient, that the solid surface around the drop was modified due to the presence of the nearby liquid. Bangham and 
Saweris [2] reported many liquids of low surface tension to incompletely spread on freshly split mica in air. To prove that there was no effect of contamination from the air they split the mica under benzene and demonstrated that benzene did not completely spread in air even following this precaution. Zisman et al. $[9,10]$ extensively studied this phenomenon and coined the term autophobic liquids, referring to liquids which are unable to spread on their own adsorbed films.

In addition, Bangham and Saweris [2] reported an interesting observation that seems to have gone unnoticed: all the single liquids which they tested did not completely spread on freshly split mica in the presence of their own saturated vapor (air being excluded). Many other liquids have recently been tested for complete spreading on clean pyrex in the presence of their saturated vapor in air [11], and most of them have indeed been shown to incompletely spread. Only very few liquids have been found to demonstrate complete spreading in the presence of their saturated vapor in air, including some of the low molecular weight paraffins : hexane, heptane, octane and decane.

Although the above mentioned experimental information can be qualitatively explained by the film pressure concept, much needs yet to be done on the quantitative level. Mostly, the effect of the thickness of the adsorbed layer on the wetting behavior and its dependence on the degree of vapor saturation need to be elucidated.

\subsection{IS THERE A COLLOIDAL FILM AT EQUILIB-} RIUM ? - The Young equation has been developed based on the assumption that the liquid-vapor interface forms a well defined angle with the solid, as shown in figure 1a. It has been hypothesized, though, that the liquid-vapor interface smoothly changes its curvature and connects to a colloidal film, as shown in figures $1 b$ and $1 c$ [12-15]. Unfortunately, no experiment has yet decisively determined which hypothesis is correct, although the limited information which exists [16] does not indicate the existence of a colloidal film extending from the liquid at equilibrium.

A thermodynamic analysis of colloidal film equilibrium $[17,18]$ reveals that the possible existence of an equilibrium situation, where a colloidal film extends from the bulk of the fluid, depends on the orientation in the gravitational field. In the absence of gravity such an equilibrium situation cannot exist. In a gravitational field, some orientations of the liquid-vapor interface may lead to an equilibrium between a colloidal film and its mother phase. The essentials of the general thermodynamic analysis are reproduced in the following, for the specific case of wetting.

As a starting point, it is assumed that the situation shown in figure $1 \mathrm{~b}$ prevails, namely, that a colloidal film extends from the bulk of the liquid. The film is sufficiently thin for colloidal interactions to be important, but also sufficiently thick to consist of two individual interfaces. It should be noted that the edge of the film is not included in the analysis. This would lead to an additional independent equilibrium condition, essentially the Young equation, which is not required for the present purpose. The mechanical equilibrium condition for this system is [18] :

$$
\int_{\Omega}\left(P_{1 \mathrm{ff}}-P_{\mathrm{v}}-2 \gamma_{\mathrm{lv}} H\right) \mathrm{d} \Omega \mathrm{d} N_{1}-\mathrm{d} U_{\mathrm{slv}}=0,
$$

where $\Omega$ is the liquid-vapor interfacial area, $P_{\text {lf }}$ is the pressure component acting on the liquid-vapor interface from the liquid side (the subscript $f$ emphasizes that part of this interface may belong to a colloidal film), $P_{\mathrm{v}}$ is the pressure in the vapor phase, $H$ is the curvature of the liquid-vapor interface, and $N_{1}$ is a displacement vector of the liquid-vapor interface pointing outwards from the liquid. $U_{\text {slv }}$ is the colloidal interaction energy, which can be written as [17] :

$$
\mathrm{d} U_{\mathrm{slv}}=-\Pi^{*} \mathrm{~d} \Omega \mathrm{d} h
$$

where $h$ is the thickness of the film and $\Pi^{*}$ is related to the disjoining pressure (19) in such a way that they are identical when the two interfaces of the film are parallel.

In the absence of gravity, equations (3) and (4) yield the following equilibrium equation :

$$
P_{\mathrm{lf}}+\Pi^{*}-P_{\mathrm{v}}-2 \gamma_{\mathrm{lv}} H=0 .
$$

This equation is essentially the same as the one developed by Deryagin et al. [12], however they assumed $P_{\mathrm{lf}}-P_{\mathrm{v}}$ to be constant. A detailed thermodynamic analysis [18] shows that $P_{\text {lf }}+\Pi^{*}$ plays in the colloidal film region the same role as the pressure in the bulk. This leads to the following condition of equilibrium between the colloidal film and the bulk from which it extends :

$$
P_{\mathrm{lf}}+\Pi^{*}=P_{1}
$$

where $P_{1}$ is the bulk pressure in the liquid. Introducing this equation into equation (5) yields the seemingly surprising result :

$$
P_{1}-P_{\mathrm{v}}-2 \gamma_{\mathrm{lv}} H=0
$$

which implies that the shape of the interface is independent of the colloidal interaction. This results should not be surprising, since the film is assumed to be sufficiently thick to consist of two individual interfaces. The important consequence of equation (7) is that the curvature of the liquid-vapor interface must be constant everywhere, since 
$P_{1}-P_{\mathrm{v}}$ is constant in the absence of gravity. This is in conflict with the change in curvature implied by the assumption that a colloidal film exists, therefore the film cannot exist at equilibrium with the bulk of the liquid. It may be of interest to notice that small changes in curvature may take place because of local variations in the surface tension, however these cannot account for the big change in curvature which should be associated with the presence of a film.

A similar analysis can be performed for the situation shown in figure 1c, where the apparent contact angle is high and a vapor film is assumed to exist. The result is the same as above : in the absence of gravity the liquid-vapor interface should meet the solid with a well defined contact angle, without a colloidal film extending from the vapor.

The above conclusions are partially modified when a gravitational field is considered. For systems such as shown in figure $1 \mathrm{~b}$, the equilibrium condition is [18] :

$$
P_{\mathrm{lf}}^{0}+\Pi^{* 0}-P_{\mathrm{v}}^{0}-2 \gamma_{\mathrm{lv}} H+\left(\rho_{1}-\rho_{\mathrm{v}}\right) g z=0,
$$

where $\rho_{1}$ and $\rho_{\mathrm{v}}$ are the densities of the liquid and vapor, respectively, $g$ is the gravitational acceleration, $z$ is a coordinate in the direction of gravity (downwards) and the superscript $o$ indicates values at $z=0$. Introducing equation (6) into equation (8) yields :

$$
P_{1}^{0}-P_{\mathrm{v}}^{0}-2 \gamma_{1 \mathrm{v}} H+\left(\rho_{1}-\rho_{\mathrm{v}}\right) g z=0 .
$$

The possible existence at equilibrium of a colloidal film depends, as discussed above, on the compatibility between the curvature variation which is implied by the assumed existence of the film and the thermodynamic equilibrium condition. Equation (9) requires the curvature to increase with $z$. This is geometrically impossible for a film extending out of a sessile drop as shown in figure $1 \mathrm{~b}$, but may be possible for a drop hanging below a solid surface. In addition, it may be possible, for example, for a liquid climbing vertically on a solid wall, as also shown in figure $1 b$.

It is hoped that methods capable of measuring the profile of the edge of the liquid with sufficient spacial resolution will be developed, so that this question can also be answered by experiment.

\section{Complete spreading.}

A spreading process which continues until a thin film is formed is called complete spreading. This happens when $\left(\gamma_{\mathrm{sv}}-\gamma_{\mathrm{sl}}\right) / \gamma_{\mathrm{lv}} \geq 1$, i.e. when $\theta=0$ or when no equilibrium contact angle is possible. This section emphasizes the experimental aspects of the effect of thin films on complete spreading, many of which are yet unexplained by theory.

Since complete spreading is characterized by the final state of a thin film, it is interesting to inquire about the thickness of this film. This question, which is meaningful only when evaporation can be eliminated, was already raised by Hardy [1] and has recently been discussed from a few points of view [20-23]. The common denominator of these analyses is the realization that the final film may, under suitable conditions, be colloidal rather than molecular. This conclusion still awaits experimental substantiation, which should not be difficult with currently available instrumentation.

\subsection{COMPLETE SPREADING OF SINGLE LIQUIDS. -} It has been known since the pioneering work of Hardy [1] that complete spreading is usually associated with spreading of a primary film ahead of the bulk of the liquid. The existence of the primary film (also sometimes called the precursor film) was demonstrated by a decrease in the friction coefficient of the solid [1], by interference patterns [2, 24], by ellipsometry $[24,25]$ and by electrical conductivity measurements [26]. The existence of such a film is thus proven by a variety of methods, however very little is known about it. To begin with, the question of the mechanism of formation is still unsettled. While Hardy believed it originates only from the vapor [1], surface diffusion was also suggested long ago [2]. It is also not clear whether complete spreading is always associated with a primary film (it is known that the reverse is not true). For example, Beaglehole [25] detected a primary film with water but not with ethanol or silicone oil. In addition, the thickness profile and its relationship to the properties of the system are largely unknown. A qualitative study by Williams [27] showed a liquid film of 1000 $2000 \AA$, which was probably the thick part of the primary film, and demonstrated the complexity of the shape of the edge of the film. There exist, however, two rough estimates for the velocity of spreading of the primary film $[25,28]$ which put it at the order of magnitude of $10 \mathrm{~cm} / \mathrm{s}$ for water on glass. These estimates were done on the basis of the time resolution of the experiments, and the actual velocities may be higher.

While not much is known about the formation and properties of the primary film itself, some observations have been made regarding its macroscopic implications on bulk spreading. Hard [1] noticed that two drops of acetic acid attracted each other when they were not more than one or two centimeters apart. While it is not absolutely clear that only a single component was involved in the experiment, since water vapor was required in the air for acetic acid to spread, it served as a dramatic demonstration of the macroscopic effect of the primary film. Marmur and Lelah [28] changed the size of the solid surface and measured its effect on the rate of spreading. They found that the rate of spreading was 
much higher for small surfaces (glass squares of $1.5 \mathrm{~cm}$ ) than for large surfaces. The effect asymptotically diminished as the surface size was increased (above $8 \mathrm{~cm}$ for water on glass squares). The dependence of the rate of spreading on the size of the solid surface is another demonstration of a macroscopic effect of the primary film, and can be explained by the following reasoning. The edge of the solid surface arrests the spreading of the primary film. Therefore, the finite size of the solid surface affects the thickness profile of the film. A gradient in the film thickness can be regarded as a gradient in the effective surface tension of the film, which influences the spreading rate.

Another way by which the primary film may be affected and thus studied is varying the vapor pressure. Data on this effect are insufficient and show conflicting trends. As mentioned above, most liquids do not completely spread under their own saturated vapor, a fact which suggests that the spreading rate decreases with increased saturation. However, measurements with water [29] indicated an increase in spreading rate with relative humidity. Since the mechanism of formation of the primary film is not yet clear, it may be premature to speculate on the explanation of these observations.

Only a few of the theoretical models which have been developed account for the primary film $[5,25$, 30,31 ]. Ruckenstein [30] seems to have been the first to include the variation in the thickness of the film in the equations for the dynamics of wetting. Teletzke et al. [31] developed a theory and presented calculations which simulated the experimental data on the effect of the size of the solid surface on the rate of spreading [28]. Recently, de Gennes [5] explained why the spreading kinetics are independent of the spreading coefficient, $\left(\gamma_{\mathrm{sv}}-\gamma_{\mathrm{sl}}-\gamma_{\mathrm{lv}}\right)$, by showing that the excess free energy is dissipated in the primary film. As pointed out by Beaglehole [25], this may not be universally true, since some of the data [29] do not support the theoretical predictions which are based on this argument [5]. Clearly, there is a need to extend the existing theories to explain the origin and formation of the primary film and its effect on the kinetics of spreading under various conditions. Especially important seems the case of volatile liquids, which has been given too little attention.

3.2 COMPLETE SPREADING OF MIXTURES. - It is very rare to encounter spreading of single liquids in practical situations. Most research studies to date, however, have employed single liquids, because of the relative simplicity of such systems. Spreading of mixtures is much more complex, since the concentrations of the components may vary spatially as well as temporally due to evaporation, diffusion or adsorption. Local gradients in concentrations induce surface tension gradients, which, in turn, interfere with the flow pattern (the Marangoni effect). Also, if one or more of the components are surface active, preferential adsorption to some of the interfaces during the process of spreading may affect its kinetics and thermodynamics. The classification of spreading as complete or incomplete is not clear-cut in the case of mixtures, since, as will be described below, initial complete spreading may turn into an incomplete one, and vice versa. Therefore, some of these phenomena will be discussed later under a separate heading.

In the simplest situations, the mode of complete spreading is retained throughout the process, but the rate of spreading is strongly influenced by the composition of the mixture. Early observations indicated that saturating water with ether or some alcohols enhanced the rate of spreading [2]. The addition of low molecular weight hydrocarbons to distilled squalane had a similar effect [24]. Pesach and Marmur [32] have recently studied the spreading of binary mixtures in a systematic manner with respect to the relative surface tensions, volatilities and individual spreading behavior of the components. Considering the Marangoni effect, which implies that liquid flows from a region of low surface tension to a region of high surface tension [33], two kinds of situations may arise, as shown in figure 2 . If $P_{\mathrm{a}}<P_{\mathrm{b}}$, where $P_{i}$ is the vapor pressure of component $i$, and $\gamma_{\mathrm{a}}>\gamma_{\mathrm{b}}$, where $\gamma_{i}$ is the surface tension of component $i$, then the higher evaporation rate of component $b$ tends to increase the surface tension at the edge of the liquid. A Marangoni flow may then be induced in the same direction as the bulk flow (Fig. 2a). In this case, the Marangoni effect is

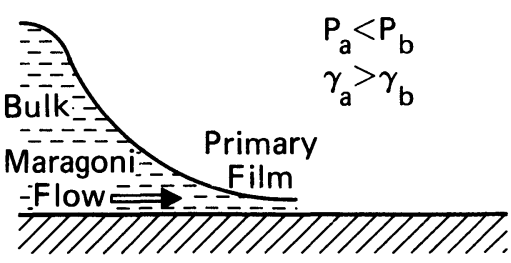

(a)

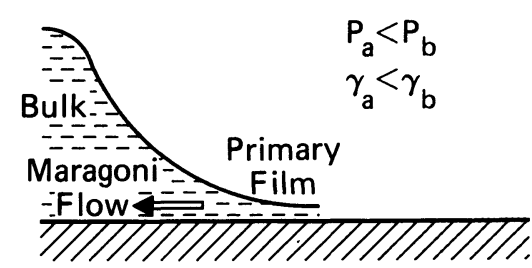

(b)

Fig. 2. - The two directions of the possible induced Marangoni flow in the case of spreading of binary mixtures. See text for explanation. 
expected to enhance the rate of spreading. Indeed, mixtures of completely spreading components, which conform with the above condition regarding the surface tensions and volatilities (such as decanehexane), have been shown to spread faster than the individual components [32].

By a similar reasoning, when $P_{\mathrm{a}}<P_{\mathrm{b}}$ and $\gamma_{\mathrm{a}}<\gamma_{\mathrm{b}}$, the spreading rate is expected to be smaller than that of the individual components, since the Marangoni induced flow is opposite to the spreading direction (Fig. 2b). An example of such a system is the mixture of acetone and octane. At relatively low concentrations of acetone (the more volatile component), the spreading rate is reduced compared with the individual components [32]. As the concentration of acetone increases, the effect becomes more dramatic: spreading is actually arrested until a sufficient amount of acetone evaporates ; only then spreading resumes.

A recent theoretical study of the Marangoni effect in spreading [34] seems to be the first in this direction. This study has concluded that surface tension gradients are important in spreading, however inadequate in overcoming adverse wettability. As will be discussed below, the latter conclusion is not supported by experiment.

\section{Mixed-mode spreading.}

As mentioned above, the composition of a mixture may change during spreading. This would vary the interfacial properties of the system and modify the final thermodynamic equilibrium. Naturally, there are two possible directions of change : (a) spreading which starts as a complete one may eventually turn into incomplete spreading ; (b) complete spreading may result from mixing of components which individually spread only incompletely. The following discussion is divided according to the nature of the observed phenomenon.

4.1 RETRACTION AND CATASTROPHIC SPREADING. - Cottington et al. [35] observed that oils with suitable additives exhibited retraction following initial spreading. Other systems in their study showed catastrophic spreading, namely very rapid spreading which formed a dendritic pattern. Marmur and Lelah [36] observed somewhat similar phenomena with aqueous surfactant solutions spreading on glass. Retraction to a situation of a non-zero contact angle followed initial complete spreading. The rate of retraction depended on the type of surfactant and was fastest for cationic ones. At high concentrations (above the CMC) branching of the drop occurred and an intricate tree pattern was formed.

The qualitative explanation of retraction seems straightforward, once the existence of a primary film is recognized. The primary film, being itself a solution of the surfactant or the additive, carries the solute and enables its adsorption on the solid ahead of the bulk of the liquid. Thus, $\gamma_{\mathrm{sv}}$ is lowered and the new surface to which the liquid is exposed is less wettable. This is supported by the observation that cationic surfactants, which adsorb very readily to the negatively charged glass surface, demonstrate retraction much faster than other types of surfactants. However, a quantitative theory for this phenomenon has not yet been developed. The mechanism underlying catastrophic spreading or branching is not clear, but these phenomena seem to occur with the same class of materials which exhibit retraction.

4.2 COMPLETE SPREADING OF MIXTURES OF NONSPREADING COMPONENTS. - Bangham and Saweris [2] noticed that some mixtures completely spread on freshly split mica although the components did not completely spread by themselves. Recently, Pesach and Marmur [32] have extensively studied this phenomenon with various binary mixtures spreading on pyrex glass. It has been shown that completely spreading mixtures of incompletely spreading components are characterized by the possibility of an induced Marangoni flow in the spreading direction, namely $P_{\mathrm{a}}<P_{\mathrm{b}}$ and $\gamma_{\mathrm{a}}>\gamma_{\mathrm{b}}$. Another interesting observation which has been made is that the effect of the concentrations of the components is not symmetrical: low concentrations of the less volatile component are sufficient to induce complete spreading, while this is not so for the other component. Eventually, the more volatile component evaporates and the drop retracts.

The explanation of this phenomenon seems to be related to the adsorbed film which has been discussed earlier. While pure liquids may be rendered autophobic by the adsorbed film, mixtures of autophobic liquids seem to behave differently. It is conceivable that a Marangoni effect takes place at the junction between the film and the drop, leading to a complete spreading situation. Clearly, this phenomenon, which may be very useful for practical applications, needs to be further studied. 


\section{References}

[1] Hardy, W. B., Philos. Mag. 38 (1919) 49.

[2] Bangham, D. H. and SAweris, Z., Trans. Farad. Soc. 34 (1938) 554.

[3] Dussan V, E. B., Ann. Rev. Fluid Mech. 11 (1979) 371.

[4] Marmur, A., Adv. Coll. Int. Sci. 19 (1983) 75.

[5] De Gennes, P. G., Rev. Mod. Phys. 57 (1985) 827.

[6] Young, T., Phil. Trans. R. Soc. (London) 95 (1805) 65.

[7] Good, R. J., Eds R. J. Good and R. R. Stromberg, (Plenum Press) Surface and Colloid Science 11 (1979) 1.

[8] Bangham, D. H. and Razouk, R. I., Trans. Farad. Soc. 33 (1937) 1459.

[9] Hare, E. F. and Zisman, W. A., J. Phys. Chem. 59 (1955) 335.

[10] Zisman, W. A., Contact Angle, Wettability and Adhesion, Adv. Chem. Ser. No. 43 Ed F. M. Fowkes (Am. Chem. Soc., 1964) p. 1.

[11] Pesach, D., M. Sc. Thesis, Technion - Israel Institute of Technology, Haifa, Israel (1985).

[12] Deryagin, B. V., Starov, V. M. and Churaev, N. V., Coll. J. U.S.R.R. (English Translation) 38 (1976) 786.

[13] Renk, F., WAYNer, P. C. Jr. and HoMsy, G. M., J. Coll. Int. Sci. 67 (1978) 408.

[14] Wayner, P. C. Jr., J. Coll. Inst. Sci. 77 (1980) 495.

[15] White, L. R., J. Chem. Soc. Farad. Trans. I, 73 (1977) 390.

[16] Oliver, J. F. and Mason, S. G., J. Coll. Int. Sci. 60 (1977) 480.

[17] Marmur, A., J. Coll. Int. Sci. 93 (1983) 18.

[18] Marmur, A., J. Coll. Int. Sci. 100 (1984) 407.
[19] Derjaguin, B. V., Coll. J. U.S.S.R (English Translation) 17 (1955) 191.

[20] Frenkel, J., Kinetic Theory of Liquids (Dover Publ., 1955), p. 324.

[21] Ruckenstein, E., J. Coll. Int. Sci. 86 (1982) 573.

[22] JoAnNy, J. F. and DE GenNes, P. G., C.R. Acad. Sci. Paris. 299 II (1984) 279.

[23] Kayser, R. F., Phys. Rev. Lett. 56 (1986) 1831.

[24] BASCOM, W. D., CotTington, R. L. and SingleterRy, C. R., in Contact Angle, Wettability and Adhesion, Adv. Chem. Ser. No. 43, Ed F. M. Fowkes (Amer. Chem. Soc., 1964) p. 355.

[25] Beaglehole, D., to be published.

[26] Ghiradella, H., Radigan, W. and Frish, H. L., J. Coll. Int. Sci. 51 (1975) 522.

[27] Williams, R., Nature 266 (1977) 153.

[28] Marmur, A. and Lelah, M. D., J. Coll. Int. Sci. 78 (1980) 262.

[29] Lelah, M. D. and Marmur, A., J. Coll. Int. Sci. 82 (1981) 518.

[30] Ruckenstein, E., Chem. Eng. Sci. 24 (1969) 1223.

[31] Teletzke, G. F., Davis, H. T. and Scriven, L. E., to be published (1987).

[32] Pesach, D. and MARMur, A., Langmuir 3 (1987) 519.

[33] LeVICH, V. G., Physico-Chemical Hydrodynamics (Prentice-Hall, Englewood Cliffs, N. J.) 1962.

[34] Neogi, P., J. Coll. Int. Sci. 105 (1985) 94.

[35] Cottington, R. L., MurPhy, C. M. and Singleterry, C. R., in Contact Angle, Wettability and Adhesion, Adv. Chem. Ser. No. 43, Ed. F. M. Fowkes (Amer. Chem. Soc., 1964) p. 341.

[36] Marmur, A. and Lelah, M. D., Chem. Eng. Comm. 13 (1981) 133. 